\title{
The Effects of Surgical Exploration on Survival of Unresectable Pancreatic Carcinoma: A Retrospective Case-Control Study
}

\author{
Daniel Åkerberg, Daniel Ansari, Roland Andersson, Bobby Tingstedt* \\ Department of Surgery, Clinical Sciences Lund, Lund University, Skåne University Hospital, Lund, Sweden \\ Email: *bobby.tingstedt@med.lu.se
}

How to cite this paper: Åkerberg, D., Ansari, D., Andersson, R. and Tingstedt, B. (2017) The Effects of Surgical Exploration on Survival of Unresectable Pancreatic Carcinoma: A Retrospective Case-Control Study. J. Biomedical Science and Engineering, 10, 1-9.

http://dx.doi.org/10.4236/jbise.2017.101001

Received: November 23, 2016

Accepted: January 16, 2017

Published: January 19, 2017

Copyright $\odot 2017$ by authors and Scientific Research Publishing Inc. This work is licensed under the Creative Commons Attribution International License (CC BY 4.0).

http://creativecommons.org/licenses/by/4.0/

\begin{abstract}
Background: Surgery may trigger the release of inflammatory factors, including cytokines, growth factors and angiogenic factors, which may stimulate tumor growth and progression. The present study was undertaken to evaluate the impact of surgical exploration on survival of patients with potentially resectable pancreatic cancer, who intraoperatively were found to have unresectable disease. Methods: Patients with pancreatic adenocarcinoma undergoing surgical exploration, without tumor resection, at our institution between 2009 and 2014, were selected and case-matched to patients who underwent primary palliative treatment. Results: A total of 116 patients were included. The surgical exploration group $(n=29)$ had significantly lower CRP levels at the time of diagnosis compared to the palliative controls $(n=87), 4$ $\mathrm{mg} / \mathrm{L}$ vs $12 \mathrm{mg} / \mathrm{L} ; \mathrm{p}=0.004$. The percentage of patients who received palliative chemotherapy was comparable between groups, but the time until start of chemotherapy was significantly longer in the surgical exploration group, 2 months vs 1 months; $\mathrm{p}<0.001$. Median survival was 7.3 months (95\% confidence interval (CI): $4.6-11.3$ months) in the surgical exploration group and 6.8 months (95\% CI: $4.7-8.6$ months) in the palliative group, $p=0.898$. In a multivariable Cox regression model, surgical exploration was not significantly associated with increased mortality risk (hazard ratio 1.06; 95\% CI: 0.69 - 1.64, $\mathrm{p}=0.782)$. Conclusion: In this case-control study, we could not detect any survival disadvantage due to surgical exploration in the setting of unresectable pancreatic cancer. Surgical exploration was associated with delayed start of palliative chemotherapy, but the percentage of patients who ultimately received chemotherapy was comparable to patients undergoing primary palliative treatment.
\end{abstract}

\section{Keywords}

Pancreatic Cancer, Surgical Exploration, Palliative Treatment, Survival 


\section{Introduction}

Pancreatic cancer has a dismal prognosis with a 5-year survival of around 5\%. It currently ranks as fourth leading cause of cancer-related death and is estimated to become the second leading cause of cancer death if no improvement is made in terms of early diagnosis and more effective treatment [1]. The incidence of pancreatic cancer varies, depending on the geographic region, but is considered to be around $10-15$ per 100,000 individuals and year [2]. The most common and also most aggressive histological subtype of pancreatic cancer is ductal adenocarcinoma, which accounts for $85 \%$ - $90 \%$ of all pancreatic cancers [3] [4].

Surgery is the only potentially curative treatment option available for pancreatic ductal adenocarcinoma. However, due to the silent nature of the disease, only $10 \%-15 \%$ are considered to be resectable at the time of diagnosis. The median survival of pancreatic cancer after curatively intended surgery is about $17-23$ months, while patients with locally advanced pancreatic cancer have a median survival of about 8 - 14 months and patients with metastatic disease have a median survival of about $4-6$ months [5]. Despite modern imaging, surgical resectability is still hard to ascertain. Up to $20 \%$ of pancreatic tumors deemed to be resectable preoperatively, may still be found be unresectable at surgery [6]

Ample research efforts have been undertaken to improve the prognosis of pancreatic cancer, but no real breakthroughs have been made. Current research focuses on characterizing the molecular pathways involved in pancreatic tumorigenesis and potential targets for intervention [7] [8]. Although the molecular mechanisms involved in pancreatic cancer development are heterogeneous and intricately regulated, growing evidence indicates that inflammation has a crucial role in tumor initiation and progression. For example, growth factor and inflammatory stimuli are required for oncogenic KRAS to induce invasive carcinoma [9]. Accordingly, clinical data suggest that patients with pancreatic cancer who display an increased systemic inflammatory state have a reduced survival rate [10] [11] [12] [13] [14]. Inflammatory cells in the vicinity of the tumor may also facilitate tumor invasiveness, contributing to local and distant tumor spread [15] [16]. However, it also seems that the process of immunosuppression is an important factor when it comes to pancreatic cancer prognosis. It has been suggested that immunosuppression, partly, trigger the systemic spread of pancreatic cancer tumour cells [17] [18]. Immunosuppression may not only be elicited by the tumor cells, but can also be iatrogenic, e.g. surgery, which may render an immunosuppressive state that can last for up to two weeks postoperatively [19] [20] [21]. Furthermore, the release of pro-inflammatory factors during surgery, such as various cytokines, growth factors and angiogenic substances, may accelerate the growth of tumor cells in the postoperative period [22].

In this study, we sought to examine whether the surgical trauma induced by operative exploration adversely affects the survival of patients with potentially resectable pancreatic cancer who are found to have unresectable disease during surgery. The control group consisted of age-matched palliative patients, with similar disease burden. 
This was a retrospective case-control study conducted at Skåne University Hospital, Lund, Sweden, between the years 2009 to 2014. Patients with pancreatic cancer were identified by performing an electronic search of the hospital medical registry using International Classification of Diseases 10th edition (ICD10) codes for tumors of the pancreas: C25.0 - C25.9 [23]. Of these patients, those with histologically proven pancreatic adenocarcinoma and tumors located in the head or body of the pancreas were identified by chart review.

Pancreatic cancer patients deemed potentially resectable at our multidisciplinary tumor conference, but were found to have unresectable disease during laparotomy, were identified. The protocol for unresectable patients were defined as metastatic disease and/or locally advanced disease. Patients undergoing primary palliative treatment were selected to serve as controls. The control patients were matched according to age at diagnosis (within 10 years), tumor size and presence of metastases. Data variables collected included age, gender, tumor size, presence of metastases, white blood cell count (WBC) levels, C-reactive protein (CRP) levels and chemotherapy details. Postoperative complications in the surgical exploration group were registered and classified according to the Clavien-Dindo system [24]. Follow-up data, including cause of death, were obtained by chart review. Institutional review board approval was obtained at Lund University to conduct this study. Missing data were not included in the material, no imputations were used.

\section{Statistical Analysis}

Continuous data are presented as median with interquartile range and categorical data as frequencies with percentage. Baseline characteristics between groups were compared using Mann-Whitney $U$ test for continuous variables, and the chi-square or Fisher's exact tests for categorical variables. Disease specific survival rates were calculated and plotted using the Kaplan-Meier method and compared with the log-rank test. Cox proportional hazard method [25] was used to adjust for possible confounding variables by developing a multivariable survival model. Factors examined included age, gender, tumor size, presence of metastases, WBC, CRP and palliative chemotherapy. All variables with a p-value < 0.25 in the univariable Cox regression analysis were selected as candidates for the multivariable analysis. In the iterative process of variable selection using forward, backward and stepwise selection, covariates were removed from the model if they were non-significant and not a confounder, as described by Hosmer-Lemeshow [26], resulting in the main effect model with no interaction terms. The data were analyzed using Stata MP 14.1 software (Stata Corp LP, College Station, TX, USA).

\section{Results}

A total of 116 patients were included in this study. Table 1 presents the baseline characteristics of the study population. The demographics in both groups were similar. Median age was 67 years in the surgical exploration group and 72 years in the palliative group. The percentage of female patients was $58.6 \%$ in the surgical 
Table 1. Baseline characteristics.

\begin{tabular}{ccccc}
\hline Variables & \multicolumn{4}{c}{ N Surgical exploration $\mathbf{N}=\mathbf{2 9}$ Palliative group $\mathbf{N}=\mathbf{8 7}$ P-value } \\
\hline Age & 116 & $67(63-77)$ & $72(64-79)$ & 0.165 \\
Female gender & 116 & $17(58.6 \%)$ & $45(51.7 \%)$ & 0.519 \\
Tumor size $(\mathrm{cm})$ & 116 & $3(2-4)$ & $3(2-4)$ & 0.658 \\
Presence of metastases & 116 & $6(20.7 \%)$ & $23(26.4 \%)$ & 0.626 \\
WBC $>10 \times 10^{9} / \mathrm{L}$ & 113 & $3(10.3)$ & $18(21.4)$ & 0.270 \\
CRP $>5$ mg/L & 112 & $12(41.4 \%)$ & $62(74.7 \%)$ & 0.001 \\
Palliative chemotherapy & 114 & $24(82.8 \%)$ & $58(68.2 \%)$ & 0.133 \\
Time to chemotherapy (months) & & $2(1-2)$ & $1(0.7-1)$ & $<0.001$ \\
No. of cycles* & & $6(3-9)$ & $5(3-6)$ & 0.377 \\
\hline
\end{tabular}

Abbreviations: CRP, C-reactive protein; $\mathrm{WBC}$, white blood cell count. *Calculated only in patients receiving palliative chemotherapy.

exploration group and $51.7 \%$ in the palliative group.

Median tumor size was $3 \mathrm{~cm}$ in both groups. Metastases were present in $6 \mathrm{pa}-$ tients $(20.7 \%)$ in the surgical exploration group and 23 patients in the palliative group (26.4\%).

The median WBC levels were $7 \times 10^{9} / \mathrm{L}\left(6 \times 10^{9} / \mathrm{L}-8 \times 10^{9} / \mathrm{L}\right)$ in the surgical exploration group and $8 \times 10^{9} / \mathrm{L}\left(6 \times 10^{9} / \mathrm{L}-10 \times 10^{9} / \mathrm{L}\right)$ in the palliative group. Three patients in the surgical exploration group had elevated WBC levels $(>10 \times$ $10^{9} / \mathrm{L}$ ) compared to 18 patients $(21.4 \%)$ in the palliative group. The median CRP levels were significantly lower in the surgical exploration group compared to the palliative group, $4 \mathrm{mg} / \mathrm{L}(3-12 \mathrm{mg} / \mathrm{L})$ vs $12 \mathrm{mg} / \mathrm{L}(5-35 \mathrm{mg} / \mathrm{L}), \mathrm{p}=0.004$. Twelve patients in the surgical exploration group hade elevated CRP levels $(>5$ $\mathrm{mg} / \mathrm{L}$ ), compared to 62 patients $(74.7 \%)$ in the palliative group, $\mathrm{p}<0.001$.

Postoperative complications occurred in 4 patients (13.8\%) in the surgical exploration group within 30 days of operation. Among these 4 patients, one developed postoperative nausea and vomiting (Clavien-Dindo grade I), the other patients developed delayed gastric emptying after the gastroenteroanastomosis and hepaticojejunostomy reconstruction (Clavien-Dindo grade II), the third patient developed enterococcal infection requiring antibiotic treatment (Clavien-Dindo grade II) and the fourth patient developed cholangitis requiring stent replacement (Clavien-Dindo grade III).

Patients received gemcitabine as a standard palliative treatment, according guidelines [27]. Palliative chemotherapy was given to 24 patients $(82.8 \%)$ in the surgical exploration group and 58 patients $(68.2 \%)$ in the palliative group. There was a significant delay to start of palliative chemotherapy for patients in the surgical exploration group compared to the palliative group, 2 vs 1 months ( $\mathrm{p}<$ 0.001). The median number of administered chemotherapy cycles was 6 in the surgical exploration group and 5 in the palliative group.

No significant differences were found between the treatment groups in the primary outcome of disease-specific survival. Figure 1 shows the Kaplan-Meier curves comparing disease-specific survival for the study groups. Median survival 
was 7.3 months (95\% confidence interval (CI): 4.6 - 11.3 months) in the surgical exploration group and 6.8 months (95\% CI: 4.7 - 8.6 months) in the palliative group, $\mathrm{p}=0.898$. Table 2 shows the univariable and multivariable analyses of the effect of surgical exploration on disease-specific survival. The unadjusted hazard ratio (HR) for surgical exploration was 1.02 (95\% CI: $0.67-1.57$; p = 0.898). In a multivariable Cox regression model adjusted for significant covariates, including gender, presence of metastases and CRP levels $>5 \mathrm{mg} / \mathrm{L}$, surgical exploration was not significantly associated with increased mortality risk (HR 1.06; 95\% CI: 0.69 - 1.64; $\mathrm{p}=0.782$ ).

\section{Discussion}

To our knowledge, this is the first study to investigate the effects of surgical exploration on survival of unresectable pancreatic cancer. Despite modern imaging, many potentially resectable pancreatic cancer patients are found to be unresectable at the time of surgery, due to either local tumor invasion or distant metastatic spread. It is hypothesized that the added trauma induced by surgery, without tumor resection, may be potentially detrimental to survival outcomes given the release of growth factors and inflammatory mediators. However, our study demonstrates that the trauma associated with explorative laparotomy does

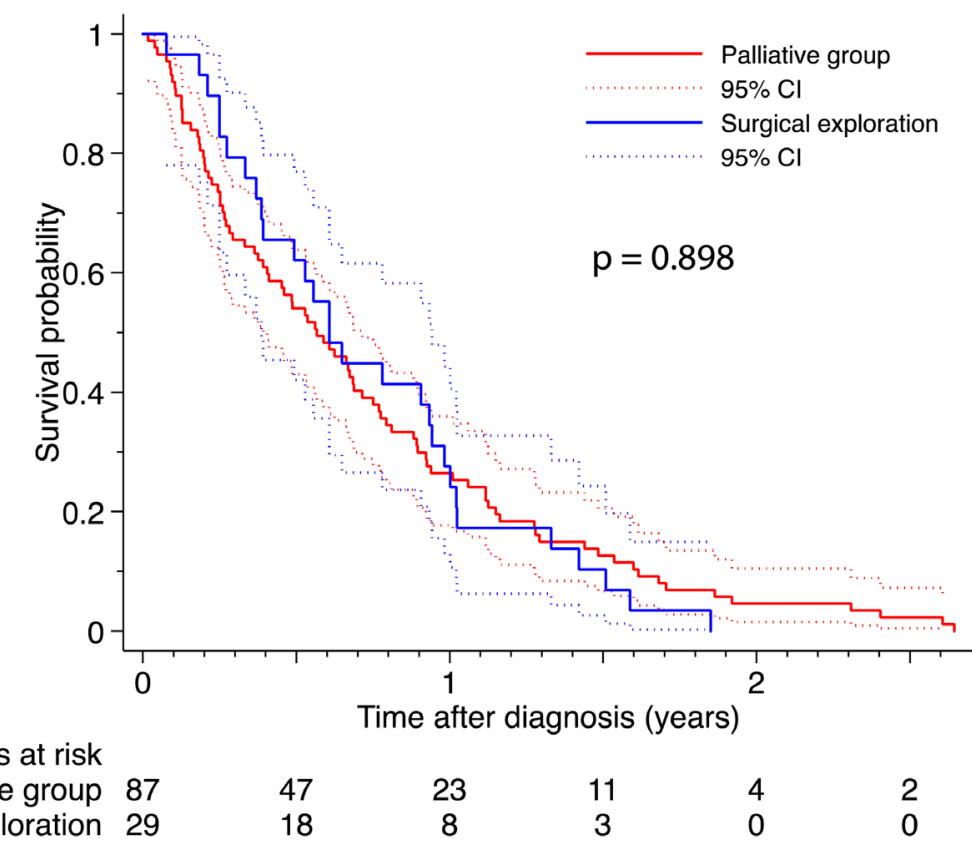

Figure 1. Comparison of survival between surgical exploration and palliative groups.

Table 2. Multivariable cox proportional hazards regression analysis.

\begin{tabular}{ccc}
\hline Variables & HR 95\% CI P-value \\
\hline $\begin{array}{c}\text { Surgical exploration vs palliative group, unadjusted } \\
\text { Surgical exploration vs palliative group, adjusted for gender, presence } \\
\text { of metastases and CRP levels }>5 \mathrm{mg} / \mathrm{L}\end{array}$ & $1.020 .67-1.570 .898$ \\
\hline
\end{tabular}

Abbreviations: CI, confidence interval; CRP, C-reactive protein, HR, hazard ratio. 
not adversely affect outcome, and survival rates were comparable to matched palliative patients not undergoing surgery. In Kaplan-Meier analysis as well as univariable and multivariable Cox regression analyses, we were unable to demonstrate a statistical difference between the groups.

The inflammatory response to surgery has many similarities to inflammatory cascades and pathways associated with tumor growth and progression. Changes in cellular components and humoral response due to inflammation have been found to stimulate tumor growth in experimental studies [28]. Furthermore, abdominal surgery is associated with a transient immunosuppression that could facilitate tumor growth and invasiveness [29]. Operative trauma to peritoneal surfaces may promote development of minimal residual disease after intraabdominal tumor resection, and the amount of tumor recurrence has been found to correlate with the degree of intraabdominal trauma in experimental studies [30].

Our secondary study objective was to elucidate the effect of surgical exploration on the initiation of palliative chemotherapy. Compared with primary palliative patients, those undergoing surgical exploration had longer time to start of chemotherapy. However, the percentage of patients that received palliative chemotherapy was comparable between groups, and the number of treatment cycles was also similar between groups. The postoperative complication rate among the surgical exploration group was low, with only 1 patient (2.6\%) developing a grade III or higher complication (cholangitis). In previous studies, postoperative complications following resectional surgery have been associated with reduced likelihood of receiving adjuvant chemotherapy, and also delays in the administration of adjuvant treatment among pancreatic cancer patients [31] [32].

Resectability of pancreatic cancer is commonly determined with computed tomography (CT) with a dedicated pancreas protocol. All patients in our study underwent $\mathrm{CT}$ preoperatively in order to accurately stage the tumors. Criteria for resectable tumors included absence of distant metastases, lack of superior mesenteric vein or portal vein distortion and clear fat planes around the celiac axis, hepatic artery and superior mesenteric artery [33]. The staging of pancreatic cancer is biased toward high specificity in order not to deny patients potentially curative resection [34]. Thus, while high positive predictive value for unresectability $(89 \%-100 \%)$, the positive predictive value of CT for resectability is much lower (45\% - 79\%) [35]. To improve resectability prediction, PET/CT, MRI and staging laparoscopy are being used as adjuncts, but CT is still the imaging modality of choice [36]. Further research is warranted in order to decrease the number of unnecessary laparotomies, e.g. through better imaging and biomarker tests.

This study was limited by the inherent nature of a retrospective review, despite careful matching of cases and controls. We adjusted for known differences between groups, but as the study was not randomized, unknown or unmeasured differences in baseline characteristics between the two cohorts might have influenced the results. 


\section{Conclusions}

In conclusion, this is the first study to report survival outcomes after "negative" explorative laparotomy in potentially resectable patients with pancreatic cancer. Although patients undergoing surgical exploration had a longer time to start of palliative chemotherapy, the percentage that received chemotherapy was similar to matched controls and, importantly, disease-specific survival rates were not significantly different. Further research in larger cohorts is needed to corroborate these findings.

\section{Summary Box}

\section{What Is Already Known:}

- Potentially resectable pancreatic cancer is sometimes found to be unresectable at the time of surgical exploration, due to local tumor invasion into adjacent large vessels or distant metastases.

- The surgical trauma induced by the explorative laparotomy itself may theoretically impair survival given the release of inflammatory factors, which may promote tumor growth and progression.

- The effects of "negative" surgical exploration on survival rates in pancreatic cancer have not been studied previously.

\section{What the New Findings Are:}

- Surgical exploration caused a delay in the start of palliative chemotherapy compared to matched palliative controls.

- However, the percentage of patients that received chemotherapy was similar between groups.

- Surgical exploration did not adversely affect survival rates.

\section{References}

[1] Rahib, L., Smith, B.D., Aizenberg, R., Rosenzweig, A.B., Fleshman, J.M. and Matrisian, L.M. (2014) Projecting Cancer Incidence and Deaths to 2030: The Unexpected Burden of Thyroid, Liver, and Pancreas Cancers in the United States. Cancer Research, 74, 2913-2921. https://doi.org/10.1158/0008-5472.CAN-14-0155

[2] Siegel, R.L., Miller, K.D. and Jemal, A. (2015) Cancer Statistics, 2015. CA-A Cancer Journal for Clinicians, 65, 5-29. https://doi.org/10.3322/caac.21254

[3] Cowgill, S.M. and Muscarella, P. (2003) The Genetics of Pancreatic Cancer. The American Journal of Surgery, 186, 279-286. https://doi.org/10.1016/S0002-9610(03)00226-5

[4] Li, D. and Jiao, L. (2003) Molecular Epidemiology of Pancreatic Cancer. International Journal of Gastrointestinal Cancer, 33, 3-14. https://doi.org/10.1385/IJGC:33:1:3

[5] Vincent, A., Herman, J., Schulick, R., Hruban, R.H. and Goggins, M. (2011) Pancreatic Cancer. Lancet, 378, 607-620. https://doi.org/10.1016/S0140-6736(10)62307-0

[6] Karmazanovsky, G., Fedorov, V., Kubyshkin, V. and Kotchatkov, A. (2005) Pancreatic Head Cancer: Accuracy of CT in Determination of Resectability. Abdominal Radiology, 30, 488-500. https://doi.org/10.1007/s00261-004-0279-Z

[7] Bailey, P., Chang, D.K., Nones K., Johns, A.L., Patch, A.-M., Gingras, M.-C., et al. 
(2016) Genomic Analyses Identify Molecular Subtypes of Pancreatic Cancer. Nature, 531, 47-52. https://doi.org/10.1038/nature16965

[8] Waddell, N., Pajic, M., Patch, A.-M., Chang, D.K., Kassahn, K.S., Bailey, P., et al. (2015) Whole Genomes Redefine the Mutational Landscape of Pancreatic Cancer. Nature, 518, 495-501. https://doi.org/10.1038/nature14169

[9] di Magliano, M.P. and Logsdon, C.D. (2013) Roles for KRAS in Pancreatic Tumor Development and Progression. Gastroenterology, 144, 1220-1229. https://doi.org/10.1053/j.gastro.2013.01.071

[10] Greer, J.B. and Whitcomb, D.C. (2009) Inflammation and Pancreatic Cancer: An Evidence-Based Review. Current Opinion in Pharmacology, 9, 411-418. https://doi.org/10.1016/j.coph.2009.06.011

[11] Fearon, K.C., et al. (2006) Definition of Cancer Cachexia: Effect of Weight Loss, Reduced Food Intake, and Systemic Inflammation on Functional Status and Prognosis. American Journal of Clinical Nutrition, 83, 1345-1350.

[12] Farren, M.R., et al. (2015) Systemic Immune Activity Predicts Overall Survival in Treatment Naive Patients with Metastatic Pancreatic Cancer. Clinical Cancer Research, 22, 2565-2574. https://doi.org/10.1158/1078-0432.CCR-15-1732

[13] Ene-Obong, A., et al. (2013) Activated Pancreatic Stellate Cells Sequester CD8+ T Cells to Reduce Their Infiltration of the Juxtatumoral Compartment of Pancreatic Ductal Adenocarcinoma. Gastroenterology, 145, 1121-1132. https://doi.org/10.1053/j.gastro.2013.07.025

[14] Tingstedt, B., et al. (2007) Predictive Factors in Pancreatic Ductal Adenocarcinoma: Role of the Inflammatory Response. Scandinavian Journal of Gastroenterology, 42, 754-759. https://doi.org/10.1080/00365520601058452

[15] Falconer, J.S., et al. (1995) Acute-Phase Protein Response and Survival Duration of Patients with Pancreatic Cancer. Cancer, 75, 2077-2082.

https://doi.org/10.1002/1097-0142(19950415)75:8<2077::AID-CNCR2820750808>3. $\underline{0 . C O ; 2-9}$

[16] Engelken, F.J., Bettschart, V., Rahman, M.Q., Parks, R.W. and Garden, O.J. (2003) Prognostic Factors in the Palliation of Pancreatic Cancer. European Journal of Surgical Oncology, 29, 368-373. https://doi.org/10.1053/ejso.2002.1405

[17] Shi, H., Li, J. and Fu, D. (2015) Process of Hepatic Metastasis from Pancreatic Cancer: Biology with Clinical Significance. Journal of Cancer Research and Clinical Oncology, 142, 1137-1161. https://doi.org/10.1007/s00432-015-2024-0

[18] Hu, H., Jiao, F., Han, T. and Wang, L.-W. (2015) Functional Significance of Macrophages in Pancreatic Cancer Biology. Tumor Biology, 36, 9119-9126. https://doi.org/10.1007/s13277-015-4127-2

[19] Ogawa, K., et al. (2000) Suppression of Cellular Immunity by Surgical Stress. Surgery, 127, 329-336. https://doi.org/10.1067/msy.2000.103498

[20] Brivio, F., et al. (2000) Surgery-Induced Decline in Circulating Dendritic Cells in Operable Cancer Patients: A Possible Explanation of Postoperative Immunosuppression. Hepatogastroenterology, 47, 1337-1339.

[21] Clark, C.E., et al. (2007) Dynamics of the Immune Reaction to Pancreatic Cancer from Inception to Invasion. Cancer Research, 67, 9518-9527.

https://doi.org/10.1158/0008-5472.CAN-07-0175

[22] Van Grevenstein, W.M.U. and Van Eijck, C.H.J. (2012) Surgical Trauma and Cancer Recurrence. In: Schwab, M., Ed., Encyclopedia of Cancer, Springer, Berlin, 3575-3578.

[23] International Classification of Diseases 10th Edition (ICD-10) Codes for Tumors of 
the Pancreas: C25.0-C25.9.

[24] Dindo, D., Demartines, N. and Clavien, P.A. (2004) Classification of Surgical Complications: A New Proposal with Evaluation in a Cohort of 6336 Patients and Results of a Survey. Annals of Surgery, 240, 205-213.

https://doi.org/10.1097/01.sla.0000133083.54934.ae

[25] Liang, K.Y., Self, S.G. and Liu, X.H. (1990) The Cox Proportional Hazards Model with Change Point: An Epidemiologic Application. Biometrics, 46, 783-793. https://doi.org/10.2307/2532096

[26] Hosmer, J.D., Lemeshow, S. and Sturdivant, R. (2013) Model-Building Strategies and Methods for Logistic Regression. In: Hosmer, D., Lemeshow, S. and Sturdivant, R., Eds., Applied Logistic Regression, 3rd Edition, John Wiley \& Sons, Hoboken, 89-94. https://doi.org/10.1002/9781118548387.ch4

[27] Bayraktar, S., Bayraktar, U.D. and Rocha-Lima, C.M. (2010) Recent Developments in Palliative Chemotherapy for Locally Advanced and Metastatic Pancreas Cancer. World Journal of Gastroenterology, 16, 673-682.

https://doi.org/10.3748/wig.v16.i6.673

[28] Ceelen, W.P., Morris, S., Paraskeva, P. and Pattyn, P. (2007) Surgical Trauma, Minimal Residual Disease and Locoregional Cancer Recurrence. Cancer Treatment and Research, 134, 51-69.

[29] Mels, A.K., et al. (2001) Immune-Stimulating Effects of Low-Dose Perioperative Recombinant Granulocyte-Macrophage Colony-Stimulating Factor in Patients Operated on for Primary Colorectal Carcinoma. British Journal of Surgery, 88, 539544. https://doi.org/10.1046/j.1365-2168.2001.01722.x

[30] Oosterling, S.J., van der Bij, G.J., van Egmond, M. and van der Sijp, J.R.M. (2005) Surgical Trauma and Peritoneal Recurrence of Colorectal Carcinoma. European Journal of Surgical Oncology, 31, 29-37. https://doi.org/10.1016/j.ejso.2004.10.005

[31] Merkow, R.P., et al. (2014) Postoperative Complications Reduce Adjuvant Chemotherapy Use in Resectable Pancreatic Cancer. Annals of Surgery, 260, 372-377. https://doi.org/10.1097/SLA.0000000000000378

[32] Wu, W., et al. (2014) The Impact of Postoperative Complications on the Administration of Adjuvant Therapy Following Pancreaticoduodenectomy for Adenocarcinoma. Annals of Surgical Oncology, 21, 2873-2881. https://doi.org/10.1245/s10434-014-3722-6

[33] National Comprehensive Cancer Network (2015) Clinical Practice Guidelines in Oncology. Pancreatic Adenocarcinoma. Version 2. http://www.nccn.org/professionals/physician gls/f guidelines.asp

[34] Wong, J.C. and Raman, S. (2010) Surgical Resectability of Pancreatic Adenocarcinoma: CTA. Abdominal Imaging, 35, 471-480. https://doi.org/10.1007/s00261-009-9539-2

[35] Brennan, D.D., et al. (2007) Comprehensive Preoperative Assessment of Pancreatic Adenocarcinoma with 64-Section Volumetric CT. Radiographics, 27, 1653-1666. https://doi.org/10.1148/rg.276075034

[36] Lopez, N.E., Prendergast, C. and Lowy, A.M. (2014) Borderline Resectable Pancreatic Cancer: Definitions and Management. World Journal of Gastroenterology, 20, 1074010751. https://doi.org/10.3748/wjg.v20.i31.10740 
Submit or recommend next manuscript to SCIRP and we will provide best service for you:

Accepting pre-submission inquiries through Email, Facebook, LinkedIn, Twitter, etc. A wide selection of journals (inclusive of 9 subjects, more than 200 journals)

Providing 24-hour high-quality service

User-friendly online submission system

Fair and swift peer-review system

Efficient typesetting and proofreading procedure

Display of the result of downloads and visits, as well as the number of cited articles Maximum dissemination of your research work

Submit your manuscript at: http://papersubmission.scirp.org/

Or contact jbise@scirp.org 